\title{
Methylenetetrahydrofolate Reductase Polymorphisms (C677T and A1298C) and Migraine Susceptibility: Correspondence
}

\author{
Rujittika Mungmunpuntipantip ${ }^{1} \cdot$ Viroj Wiwanitkit $^{2}$
}

Received: 29 January 2022/ Accepted: 21 February 2022/Published online: 5 March 2022

(C) The Author(s), under exclusive licence to Association of Clinical Biochemists of India 2022

Dear Editor, we would like to comment on the publication "Relation Between Methylenetetrahydrofolate Reductase Polymorphisms (C677T and A1298C) and Migraine Susceptibility [1]". Rai and Kumar concluded that "Results of present meta-analysis indicate overall association between MTHFR C677T polymorphism with migraine .......not show any association with Caucasian population and $M O$ cases [1]". This meta-analysis is interesting and can give a clue of clinical association of the studied polymorphisms. However, for a meta-analysis, it is usually hard to control confounding factors. It cannot control the confounding factor in each recruited study. An important concern is effect of other confounding genetic polymorphisms that might affect migraine susceptibility. Examples of those polymorphisms are PRDM16, LRP1 and TRPM8 and TNFalpha gene polymorphism [2,3]. The pattern of those polymorphisms might be different in different setting of recruited reports in the meta-analysis. This might be an explanation on the observation on heterogenous association in the present report.

\section{Declarations}

Conflict of interest The authors declare that they have no conflict of interest.

\section{References}

1. Rai V, Kumar P. Relation between methylenetetrahydrofolate reductase polymorphisms (C677T and A1298C) and migraine susceptibility. Ind J Clin Biochem. 2022;37:3-17. https://doi.org/ 10.1007/s12291-021-01000-0.

2. Kesavan P, Satheesh AP, Husain RSRA, Veerappan U, Kannaian S, Ahmed SS, Veerabathiran R. Genetic predisposition of TNFalpha gene polymorphism in South-Indian Migraineurs and metaanalysis. Front Biosci (Elite Ed). 2021;13(2):226-36.

3. Zafar R, Saleem T, Sheikh N, Maqbool H, Mukhtar M, Abbasi MH. PRDM16, LRP1 and TRPM8 genetic polymorphisms are risk factor for Pakistani migraine patients. Saudi J Biol Sci. 2021;28(10):5793-9.

Publisher's Note Springer Nature remains neutral with regard to jurisdictional claims in published maps and institutional affiliations.
Rujittika Mungmunpuntipantip rujittika@gmail.com

1 Private Academic Consultant, Bangkok 101102, Thailand

2 Dr DY Patil University, Pune, India 